\title{
Study of CuAlBe Shape Memory Alloy by X-Ray Diffraction
}

\author{
F. Moreau, A. Tidu, Ph. Barbe*, A. Eberhardt* and J.J. Heizmann \\ CMS/LMPC/ISGMP, Faculté des Sciences, Ile du Saulcy, 57045 Metz cedex 01, France \\ * LPMM/ISGMP, Faculté des Sciences, Ile du Saulcy, 57045 Metz cedex 01, France
}

A complete study of $\mathrm{Ms}-30^{\circ} \mathrm{C}$ polycrystalline CuAlBe shape memory alloy is made by $\mathrm{X}$ Ray diffraction using a texture goniometer equipped with a curved position sensitive detector. Austenite and martensite unit cells parameters are measured. Measured indexes and orientation of the habit plane are compared to those expected by the lattice parameters. Austenite after extrusion shows a strong fiber texture, volumic fraction of austenite and martensite are measured according to the deformation. Internal deformations of three grains of a $\mathrm{Ms}-100^{\circ} \mathrm{C} \mathrm{CuAlBe}$ sample are followed during their macroscopic deformation.

\section{INTRODUCTION}

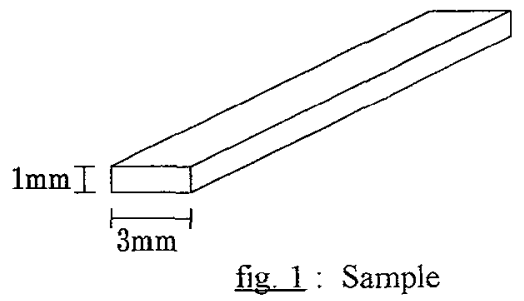

The composition of CuAlBe alloy in weight is: $87.2 \%$ of Copper , $12.3 \%$ of Aluminium and $0.52 \%$ of Beryllium. It is in austenitic phase at room temperature. The unit cell is $\mathrm{DO}_{3}$ with a cell parameter of $0.582 \mathrm{~nm}$. Martensitic transformation starts at $-30^{\circ} \mathrm{C}$ and give a monoclinic $18 \mathrm{R}$ martensite, the unit cell parameters measured by using $\mathrm{X}$-ray diffraction patterns are: $a^{\prime}=0,446 \mathrm{~nm} \quad b^{\prime}=0,522 \mathrm{~nm} \quad c^{\prime}=1,275 \mathrm{~nm} \beta=83,3^{\circ}$ (1). The grain size is about 50 to $80 \mu \mathrm{m}$. The alloy is extruded and supplied by Tréfimétaux (France).
The texture goniometer used is a Multipole Figure Goniometer (2) equipped with a $9 \mathrm{~kW} \mathrm{Fe}$ rotating anode powered. $\mathrm{K}$ $\alpha 1$ and $K \alpha 2$ are not separated, the average wavelength is $19.37 \mathrm{~nm}$. The curved position sensitive detector measures simultaneously diffracted beams on a $120^{\circ}$ angular range with a $0.03^{\circ}$ resolution.

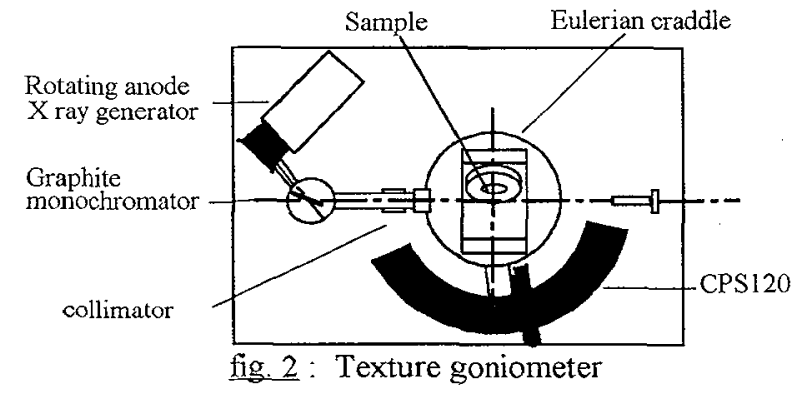




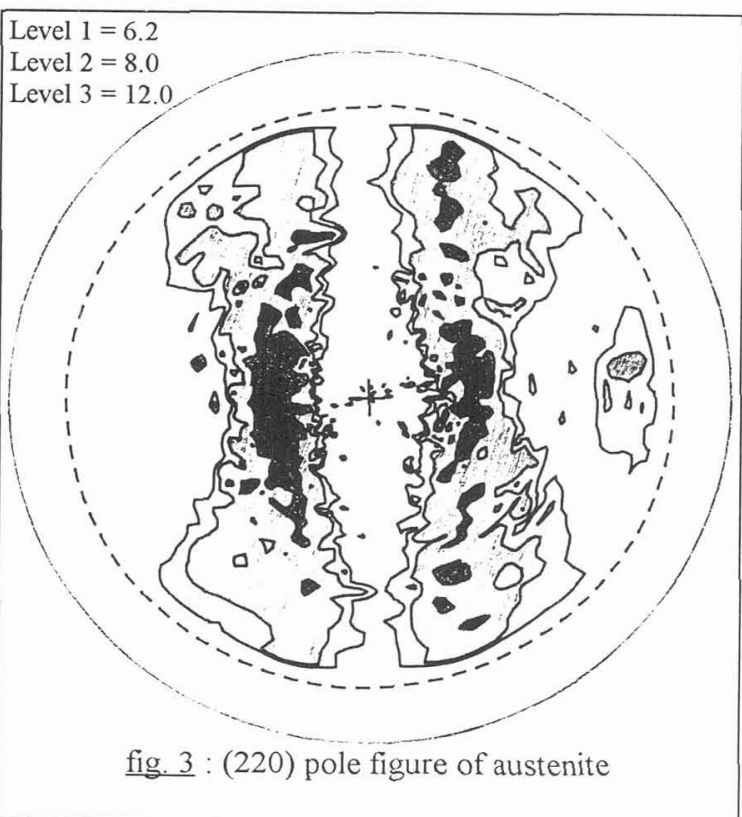

\section{TEXTURE}

Extrusion process induces a texture. This texture is the same between the surface and the core of the sample (verified by experiment). The sample has large grains, so, it is impossible to obtain the texture with one pole figure. We added 6 pole figures from large areas to obtain a good representation of texture (fig.3).

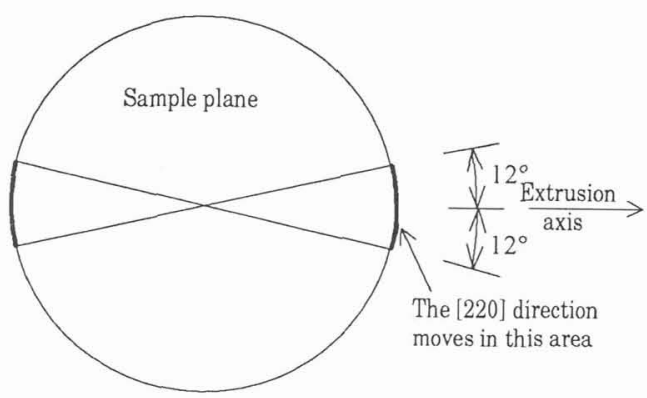

fig. 4 : Orientation of the fiber axis

The (220) pole figure presented on fig. 3 shows a strong fiber texture which [110] fiber axis can move \pm $12^{\circ}$ from the extrusion axis in the plane of the sample (fig 4 ).

Wire extrusion or wire drawing processes of $\mathrm{BCC}$ materials leads to a [110] fiber texture parallel to the deformation direction, the observed angular spread of this fiber axis could be explained by the rectangular shape of the extrusion die. This texture is used to calculate the Schmidt factors for modelization of CuAlBe shape memory alloys behaviour.

\section{AUSTENITE AND MARTENSITE VOLUMIC FRACTION MEASUREMENT ACCORDING TO THE DEFORMATION}

The quantitative phase analysis use intensities of diffraction peaks of each phase randomly oriented. Using the goniometer, the diffraction patterns integrated on the major part of the pole figure give also a randomly oriented sample even if a strong texture is present. On the X-ray diffraction patterns, from $48.5^{\circ}$ to $64^{\circ}(2 \theta)$ we have several peaks of the martensitic phase (fig. 7c). The more intense austenite peak is observed at $56.2^{\circ}$ (fig. 7a). Unfortunately all these peaks are mixed. To obtain the peak integrated intensity of each phase, a fitting procedure is used.

The theoretical integrated intensity from $48.5^{\circ}$ to $64^{\circ}$ of the martensitic phase is 172.81 and the one of the austenite peak is 182.81 (in arbitrary unit) (1). These values are verified by experiment.

$$
X m=\frac{\frac{I_{m}}{R_{m}}}{\frac{I_{a}}{R_{a}}+\frac{I_{m}}{R_{m}}} \Rightarrow X m=\frac{I_{m}}{I_{m}+I_{a} \frac{R_{m}}{R_{a}}}
$$

Xm : Volumic fraction of martensite

Im : Integrated intensity of the martensite phase

Ia : Integrated intensity of the austenite peak.

$\mathrm{Rm}=172.81$ : Theoretical integrated intensity of the martensite phase

$\mathrm{Ra}=182.81$ : Theoretical integrated intensity of the austenitic phase 
To take into account texture and heterogeneity of the sample, we measure the $\mathrm{X}$-ray diffraction patterns integrated on a great part of the pole figure.

The $7 \mathrm{a}, 7 \mathrm{~b}, 7 \mathrm{c}$ figures show the evolution of the X-ray diffraction pattern according to the deformation:

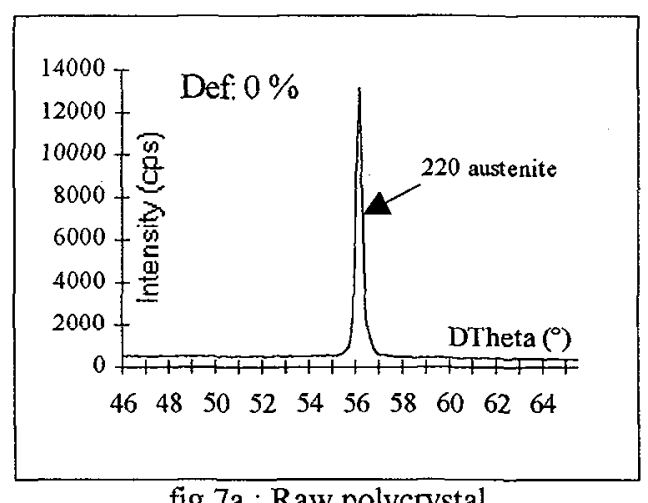

fig 7a : Raw polycrystal

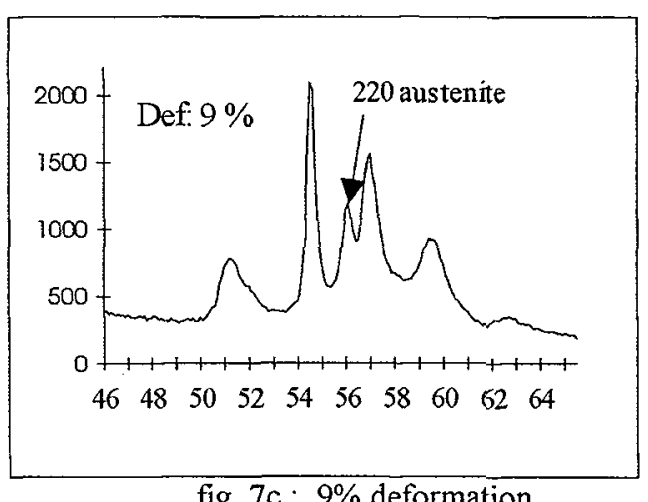

fig. $7 \mathrm{c}: 9 \%$ deformation

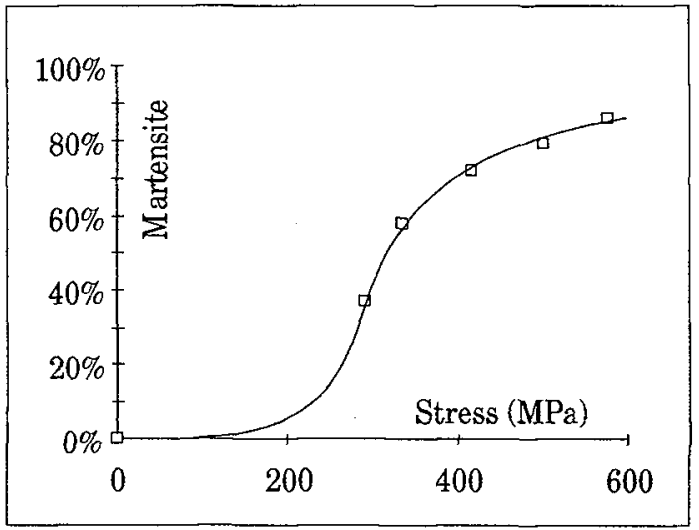

fig. 8b : Martensitic fraction against stress

fig. 8a : Volumic fraction of martensite against deformation

These results on the evolution of the volumic fraction of martensite according to stress (fig. $8 \mathrm{~b}$ ) are in agreement with those measured by electrical resistance on $\mathrm{CuZnAl}$ (3) 
The mean error on the volumic fraction of the martensitic phase is about $5 \%$. It is essentially induced by uncertainty of the calculated peak intensities.

Theoretical explanations of the behaviour of the curve (8a) are now difficult, because, intuitively, it seems that the concavity must be in opposite side. Nevertheless, this behaviour could be explained by:

- The formation of a new martensite (6R), not yet observed by X-ray diffraction on this kind of polycrystalline sample.

- Plastification of the martensite.

- A free surface effect.

\section{$42^{\text {nd }}$ AND $3^{\text {rd }}$ ORDER STRAINS IN A CuAIBe POLYCRYSTAL}

The evolution of $2^{\text {nd }}$ and $3^{\text {rd }}$ order strains $(4)$ is followed in three grains of the same sample according to macroscopic deformation applied to the sample.

The analysed sample is a Ms $-100^{\circ} \mathrm{C}$ CuAlBe ribbon of $3 \times 1 \mathrm{~mm}$ cross section. The average grain size is $0.5 \mathrm{~mm}$.

Three grains of different orientations are chosen from the austenite (220) pole figure. Successive loadings are made on the sample. After each of them, the position of diffraction peak gives the average grain deformation and its Fourier analysis, by the Warren-Averbach method (5), gives the mean particle size and the residual mean square strains $\left\langle\varepsilon^{2}>\right.$.
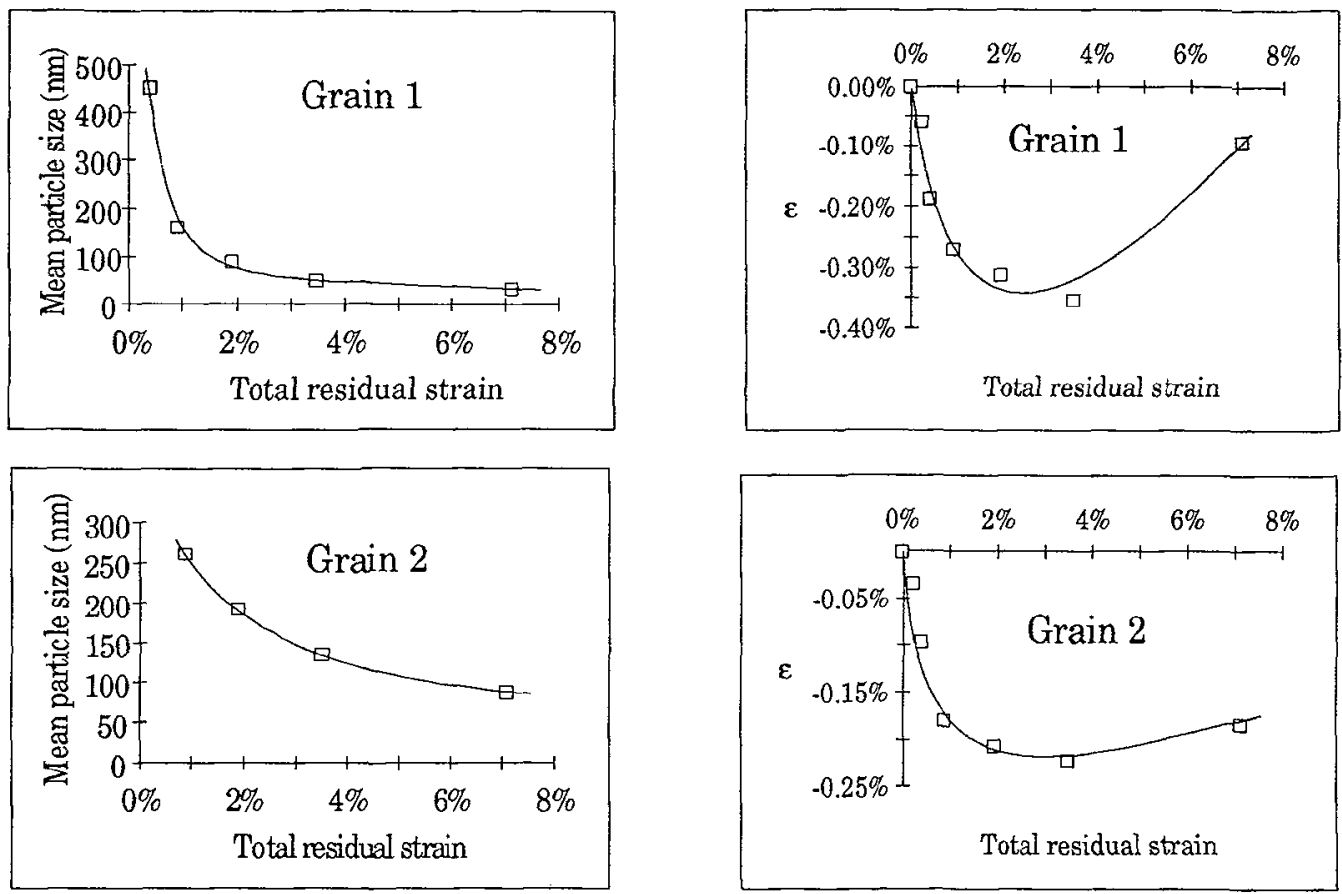


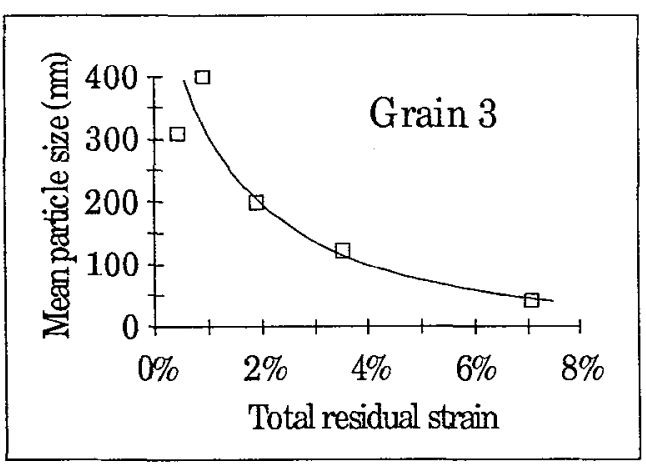

$3^{\text {rd }}$ order strain

During deformation, the grain is divided in more and more small domains. The domain size is linked to the density of defects in the grain (dislocations...).

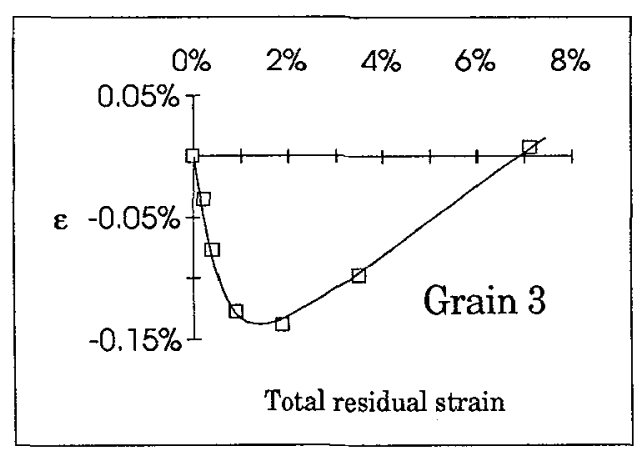

$2^{\text {nd }}$ order strain

$\varepsilon$ is the average strain of the grain measured by X-ray diffraction. The total residual strain is the deformation of the sample.

During deformation, the austenite grains are sliced by martensite plates but this slicing don't explain the smallness of the mean domain size obtained ( $40 \mathrm{~nm}$ for a grain size of $0.5 \mathrm{~mm}$ ). Now, we are not abble to explain that the strain in austenite begins to decrease at $3 \%$ of macroscopic deformation.

These results show that there is a great heterogeneity of the strain from grain to grain.

However with the Warren-Averbach method, it is impossible to see if the broadening of the diffraction peak comes from an heterogeneity in the deformation of a grain or from a $3^{\text {rd }}$ order strain.

\section{MEASUREMENT OF THE HABIT PLANE ORIENTATION}

The analysed sample is a $\varnothing 1.2 \mathrm{~mm}$ single crystal wire made by LPMM by melting of $\mathrm{Cu}(87.2) \mathrm{Al}(12.3)$ $\mathrm{Be}(0,52), \mathrm{Ms}-30^{\circ} \mathrm{C}$, alloy austenitic at room temperature. Melting induce an evaporation of Aluminium and Beryllium which results in a martensitic transformation starting point about $0^{\circ} \mathrm{C}$.

The wire is coated with resin. By polishing, we perform two perpendicular sides which are used for the sample positioning on the goniometer.

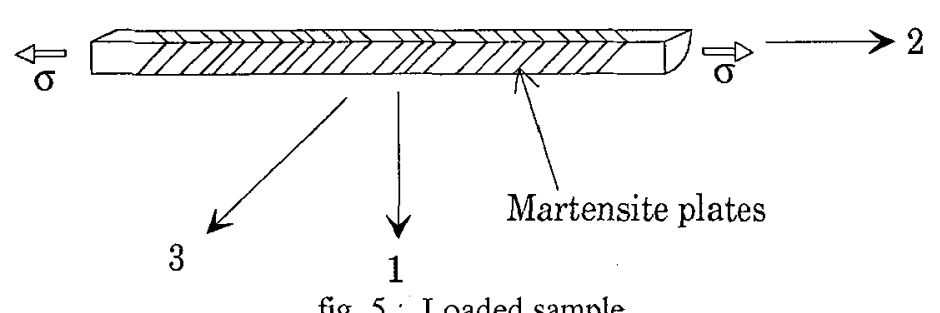

fig. 5 : Loaded sample
With a pole figure, the single crystal orientation is determined. The sample is removed from the resin block. With a little traction device the wire is loaded to create several martensite plates.

Only one variant appears during the loading , but it is possible to see one or two other variants for particular orientations of the crystal according to the loading direction. Martensite plates give lines one each side of the sample. The vector product of the two traces gives the habit plane orientation. The habit plane indexes are given with respect to the austenite lattice. 
Calculation of habit plane orientation originally developed by Suzuki (6) from austenite and martensite unit cell parameters (given above) gives ( $\begin{array}{lll}1 & 3.7 & 4\end{array}$ ) as indexes of the habit plane.

Three measurements from 3 pieces coming from the same single-crystal have been carried out and the results are the following: ( 14.04 .1$)$ ( $\left.\begin{array}{lll}1 & 4.2 & 4.3\end{array}\right)$ ( 13.94 .5 ).

The average orientation is ( $\left.\begin{array}{lll}1 & 4.1 & 4.2\end{array}\right)$.

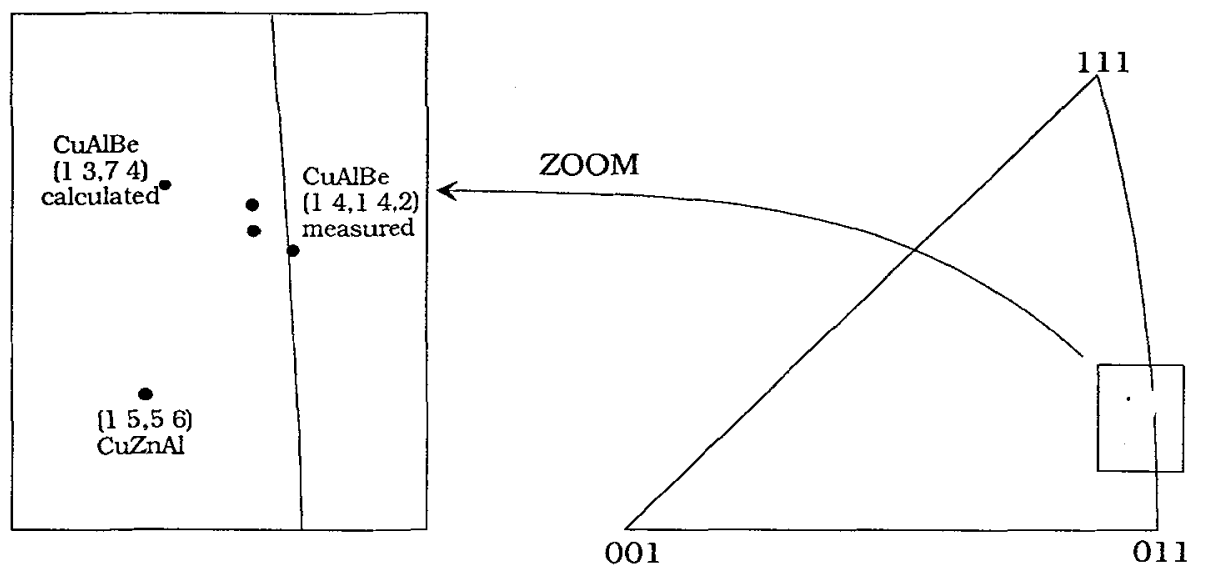

fig. 6 : Representation in the austenite standard triangle:

The angle between the calculated habit plane and the average measured one is $1.7^{\circ}$. This small difference is explained either by the unit cells parameters which are not known very accurately or by the precision of measurement. Taking into account these small errors, there is a very good agreement between experimental and calculated orientation of the habit plane..

\section{CONCLUSION}

$\mathrm{X}$ ray diffraction is a good technique to analyse special materials as shape memory alloys. The main difficulty is that all the informations given by the material are mixed, our study is to identify and to separate them. $\mathrm{X}$ ray diffraction allows to know and describe the evolution of several parameters as stresses, for example, which occur during the phase transformation. This knowledge is necessary for a better understanding of the mechanisms involved in the martensitic transformation.

\section{References}

(1) F. Moreau, A. Tidu To be published

(2) J. J. Heizmann, C. Laruelle and A. Vadon, Analysis, 16, n6, 334-340 (1988)

(3) Christian Lexellent \& Pierre Vacher "Modeling of the pseudo-elastic behavior of polycrystalline shape memory alloys CuZnAl" Proceedings of plasticity '91 p508

(4) Maeder G. Ann. Chim. Fr. (1986), 11, 159-175

(5) Warren Averbach "Local atomic arrangements studied by X ray diffraction" Edited by J.R. Cohen \& J.E. Hilliard 1965

(6) Setsuo Kajiwara, Trans. JIM, Vol. 17, 1976 